\title{
ESTRATEGIAS DE AFRONTAMIENTO Y CRECIMIENTO POSTRAUMÁTICO EN MUJERES CON Y SIN CÁNCER DE MAMA
}

\author{
Lucía Morales-Sánchez \\ Universidad de Cádiz. Departamento de Psicología. Laboratorio de Inteligencia Emocional \\ Puerto Real, España \\ lucia.morales@uca.es \\ Paloma Gil-0larte \\ Universidad de Cádiz. Departamento de Psicología. Laboratorio de Inteligencia Emocional \\ Puerto Real, España \\ Instituto para el Desarrollo Social Sostenible (INDESS) \\ Universidad de Cádiz \\ Rocío Gómez-Molinero \\ Universidad de Cádiz. Departamento de Psicología. Laboratorio de Inteligencia Emocional \\ Puerto Real, España \\ Rocío Guil \\ Universidad de Cádiz. Departamento de Psicología . Laboratorio de Inteligencia Emocional \\ Puerto Real, España \\ Instituto para el Desarrollo Social Sostenible (INDESS) \\ Universidad de Cádiz
}

Fecha de Recepción: 15 Marzo 2019

Fecha de Admisión: 30 Abril 2019

\section{RESUMEN}

Entendemos por Crecimiento Postraumático (CPT en adelante) el cambio positivo que un individuo puede llegar a experimentar tras un suceso traumático. Padecer cáncer de mama constituye una experiencia traumática, tanto por el impacto del diagnóstico, como por los tratamientos administrados. No obstante, toda persona vivencia momentos traumáticos a lo largo de sus vidas, pudiendo obtener a partir de ellos ciertos beneficios y/o CPT, o no. Consideramos que el desarrollo de CPT a partir de experiencias traumáticas puede verse promovido, o dificultado, por el uso de diferentes estrategias de afrontamiento (EA en adelante). Es por ello que en el presente estudio nos planteamos como objetivos: 1) comprobar si existen diferencias estadísticamente significativas en Ios niveles de CPT y las EA empleadas entre mujeres con cáncer de mama y sanas, 2) explorar la existencia de correlaciones estadísticamente significativas entre las EA empleadas y el nivel de CPT; y 3) analizar la posible capacidad explicativa y predictiva del uso de diversas EA y el desarrollo de CPT, controlando el posible efecto de la edad y la enfermedad. 


\section{ESTRATEGIAS DE AFRONTAMIENTO Y CRECIMIENTO POSTRAUMÁTICO EN MUJERES CON Y SIN CÁNCER DE MAMA}

Participaron un total de 110 mujeres, 45 con cáncer de mama (40.9\%) y 55 sin cáncer (59.1\%). Se administraron el Inventario de Crecimiento Postraumático (PTGI) de Tedeschi y Calhoum (1996) y la versión española del Coping Strategies Inventory (Cano y cols, 2007). Los resultados informan que ambos grupos de mujeres se diferenciaron en el uso de la EA expresión de emociones; que el CPT correlaciona con distintas EA, siendo las EA apoyo social y la resolución de problemas las que explican y predicen el CPT, con independencia de la edad y la enfermedad. Concluimos en la necesidad de que ambas EA deberían ser incorporadas en intervenciones dirigidas a la promoción del CPT en mujeres que han padecido una experiencia traumática, sea esta clínica o no.

Palabras clave: crecimiento postraumático; estrategias de afrontamiento; resolución de problemas; apoyo social; mujeres; cáncer de mama

\section{ABSTRACT}

Coping strategies and Post-Traumatic Growth in women with and without breast cancer. PostTraumatic Growth (PTG now on) can be understood as the positive change that an individual experiences as a result of a traumatic event. Breast cancer can be considered as a traumatic situation due both to the diagnosis impact and to the administered treatments. However, regardless of suffering a serious illness, all people face traumatic situations in their lives through which they can obtain -or not- certain benefits and/or PTG. Likewise, the experimented growth derived from these events could be promoted or hindered by the use of different coping strategies (CS from this point on). In this sense, the objectives of this study are: 1) to examine whether there are statistically significant differences in the levels of PTG and the CS used among women with and without breast cancer, 2) to explore the existence of statistically significant correlations between the used CS and the levels of PTG; and 3) to analyze the prospective explanatory and predictive capacity of the use of various CS and the development of PTG, controlling for the possible effect of age and the disease.

The sample was comprised of 110 women, 45 of them with breast cancer $(40.9 \%)$ and 55 without cancer $(59.1 \%)$. Two instruments were administered: The Post-Traumatic Growth Inventory (PTGI) (Tedeschi and Calhoum, 1996) and the Spanish version of the Coping Strategies inventory (Cano et al, 2007). The results reported that both groups differed in the use of CS emotional expression; that PTG correlates with different CS, being social support and problems solving those CS that explain and predict the PTG, regardless of age and illness. We conclude that both CS should be included in interventions aimed at promoting PTG in women who have suffered a traumatic experience, being this clinic or not.

Keywords: post-traumatic growth; coping strategies; problem solving; social support; women; breast cancer

\section{ANTECEDENTES}

La enfermedad que causa un mayor número de muertes en el siglo XXI, erigiéndose por tanto como uno de los grandes problemas de salud pública es el cáncer (Guil, Zayas, Gil-Olarte, Guerrero, González \& Mestre, 2016). Según indica la Sociedad Española de Oncología Médica (SEOM, 2016), la neoplasia más común en las mujeres españolas actualmente, así como de los países industrializados, y primera causa de muerte por tumor en mujeres de 35 a 50 años, es el cáncer de mama (Cerezo, Ortiz-Tallo y Cardenal, 2009). Por otro lado, en las últimas décadas, y enmarcándose dentro del paradigma de la Psicología Positiva, han aumentado los estudios de enfermedades desde una perspectiva salutogénica, desde los que se exploran diferentes variables psicológicas, sociales y emocionales que ayudan a prevenir la aparición de trastornos psicopatológicos derivados del diagnóstico de una enfermedad, o de experimentar situaciones traumáticas, y que promuevan al mismo tiempo mejoras en la calidad de vida y el bienestar de las personas (Guil et al., 2016). 
El diagnóstico y tratamiento de cáncer de mama supone indiscutiblemente un acontecimiento traumático para las personas que lo padecen (Cano, 2005; Guil, Zayas, Gil-Olarte, Guerrero \& Mestre, 2017). No obstante, existen innumerables situaciones y experiencias que pueden resultar traumáticas, tras las cuales las personas consiguen obtener un cambio positivo (Zayas et al, 2018). En este sentido, crisis comunes como enfermedades físicas, duelos, divorcios y eventos traumáticos tales como desastres naturales y guerras, dan forma a la vida de las personas de manera única y duradera (Schaefer y Moos, 1998).

Centrándonos en estos cambios positivos experimentados como resultado de vivir situaciones traumáticas o estresantes, el término Crecimiento Postraumático (CPT en adelante), hace referencia a la "percepción de los cambios positivos que resultan del esfuerzo personal tras una crisis vital 0 acontecimiento traumático" (Tedeschi y Calhoum, 1995). Este cambio supone una transformación personal en la forma de responder a las demandas vitales que integra un bienestar no apreciado antes de ocurrir el suceso traumático y que se encuentra estrechamente relacionado con la forma de afrontar el estrés (Calhoum y Tedeschi, 2006). De esta forma, siguiendo a Prati y Pietrantoni (2009), consideramos que el proceso de CPT influido y determinado por una gran variedad de factores que pueden promover o dificultar este crecimiento, entre los cuales se encuentran las estrategias de afrontamiento y el apoyo social.

El afrontamiento (del inglés coping), hace referencia a la respuesta cognitiva y de comportamiento que los individuos usan para manejar o tolerar el estrés, (Lazarus y Folkman, 1984 en Tobin et al., 1989), o situaciones como el proceso de adaptación a un diagnóstico y/ o tratamiento de cáncer (Barroilhet et al., 2015). Consideramos, por tanto que, dado que el afrontamiento de la adversidad se considera como un aspecto muy relevante del funcionamiento personal (Fierro, 1993 en Cano et al., 2007), las estrategias de afrontamiento deben actuar como un factor clave en el proceso de CPT. En este sentido, siguiendo a Prati y Pietrantoni (2009), tanto las estrategias de afrontamiento como el apoyo social, contribuyen al crecimiento obtenido tras un hecho traumático. Por su parte, el modelo afrontamiento de Schaefer y Moos (1998) considera al apoyo social un recurso ambiental clave para comprender los resultados positivos producto de las crisis vitales. En la misma línea, Tedeschi y Calhoun (2004) incluye en su modelo de CPT al apoyo social como predictor de cambios positivos tras eventos traumáticos.

Con independencia de padecer una enfermedad o no, todas las personas pueden obtener cambios positivos tras experimentar situaciones traumáticas o estresantes a lo largo de sus vidas. Por ello nos planteamos en este estudio la necesidad de analizar si se encuentran diferencias estadísticamente significativas en el CPT de mujeres que padecen cáncer de mama, en comparación con un grupo de mujeres que sin esta enfermedad. Además, la escasez de estudios realizados hasta el momento que analicen el impacto del uso de diferentes estrategias de afrontamiento sobre el crecimiento postraumático convierten esta investigación en un punto de inflexión que da paso al inicio de estudios que aporten valor a la mejora de la calidad de vida y el bienestar de mujeres, ya sea en ámbitos clínicos como no clínicos.

\section{OBJETIVOS}

Los objetivos del presente estudio son los siguientes:

Analizar si existen diferencias intergrupales entre las estrategias de afrontamiento y el CPT de mujeres con cáncer y sin cáncer.

Explora la existencia de relaciones estadísticamente significativas entre las estrategias de afrontamiento y el CPT.

Explorar la capacidad explicativa y predictiva de diversas estrategias de afrontamiento en el Crecimiento Postraumático controlando la influencia de padecer o no cáncer y la edad. 


\section{ESTRATEGIAS DE AFRONTAMIENTO Y CRECIMIENTO POSTRAUMÁTICO EN MUJERES CON Y SIN CÁNCER DE MAMA}

\section{MÉTODO}

\section{Muestra}

Las participantes de esta investigación fueron 110 mujeres divididas en dos grupos: uno, formado por 45 mujeres supervivientes de cáncer de mama (40.9\%) y otro constituido por 55 mujeres que no han padecido esta enfermedad (59.1\%). Todas son naturales de la provincia de Cádiz y con respecto a su edad, la media del primer grupo es de $52.20(D T=8.85)$ y la del segundo de 38.78 $(D T=10.41)$.

\section{Metodología e instrumentos}

Se administraron dos instrumentos: el Inventario de Crecimiento Postraumático (PTGI) (Tedeschi y Calhoum, 1996) y la versión española del Coping Strategies Inventory (CSI) (Cano y cols, 2007).

Por una parte, el Inventario de Estrategias de Afrontamiento es un cuestionario cuyo fin reside en conocer el tipo de situaciones que causa problemas a las personas en su vida cotidiana y cómo estas se enfrentan a dichos problemas. Es una escala de 40 ítems con formato de respuesta tipo Likert, con un rango de 0 (No se utiliza) a 20 (Se utiliza mucho) a través de los cuales se obtiene información sobre ocho dimensiones/estrategias de afrontamiento diferenciadas (Nava-Quiroz et al., 2010): 1) Resolución de problemas (estrategias cognitivas y conductuales encaminadas a eliminar el estrés modificando la situación que lo produce), 2) Reestructuración cognitiva (estrategias cognitivas que modifican el significado de la situación estresante), 3) Apoyo social (estrategias referidas a la búsqueda de apoyo emocional), 4) Expresión emocional (estrategias encaminadas a liberar las emociones que acontecen en el proceso de estrés), 5) Evitación de problemas (estrategias que incluyen la negación y evitación de pensamientos 0 actos relacionados con el acontecimiento estresante), 6) Pensamiento desiderativo (estrategias cognitivas que reflejan el deseo de que la realidad no fuera estresante), 7) Retirada social (estrategias de retirada de amigos, familiares, compañeros y personas significativas asociadas con la reacción emocional en el proceso estresante) y 8) Autocrítica (estrategias basadas en la autoinculpación y la autocrítica por la ocurrencia de la situación estresante o su inadecuado manejo).

Por otra parte, el Inventario de Crecimiento Postraumático (PTGI, Posttraumatic Growth Inventory, Calhoun y Tedeschi, 2006; Tedeschi y Calhoun, 1996), es un cuestionario de 21 ítems que mide la percepción de beneficios personales en personas que han pasado por un acontecimiento traumático. Este cuestionario está constituido por cinco dimensiones: 1) Relación con los demás, 2) Nuevas posibilidades, 3) Fuerza personal, 4) Cambio espiritual, y 5) Apreciación por la vida. Tiene un formato de respuesta de tipo Likert, con un rango de puntuación de 0 (ningún cambio) a 5 (un cambio muy importante) en sentido positivo: a mayor puntuación, mayor cambio percibido (CostaRequena y Gil, 2007). En este estudio utilizaremos la dimensión total de Crecimiento Postraumático.

\section{ANÁLISIS Y RESULTADOS}

A fin de dar respuesta a los objetivos de nuestro estudio se analizaron, en primer lugar, los valores descriptivos de la muestra total, como del grupo de mujeres con y sin cáncer (Ver tabla 1). Los resultados mostraron que las mujeres con cáncer de mama presentan una media más alta en las estrategias resolución de problemas, reestructuración cognitiva, evitación de problemas y en el CPT. Por el contrario, las mujeres sin cáncer puntúan más alto en las estrategias autocrítica, expresión de emociones, pensamiento desiderativo, apoyo social, y retirada social. 
Tabla 1. Análisis descriptivos de la muestra total, mujeres con cáncer y mujeres sin cáncer de mama, rango, y alfas de Cronbach

\begin{tabular}{|c|c|c|c|c|c|c|c|c|}
\hline \multirow[b]{2}{*}{$\begin{array}{c}\text { Variabl } \\
\text { es }\end{array}$} & \multirow[b]{2}{*}{$\begin{array}{c}\text { Rang } \\
\text { o }\end{array}$} & \multicolumn{2}{|c|}{$\begin{array}{r}\text { Muestra } \\
\text { total }\end{array}$} & & \multicolumn{2}{|c|}{$\begin{array}{l}\text { Mujeres con } \\
\text { cancer }\end{array}$} & \multicolumn{2}{|c|}{$\begin{array}{c}\text { Mujeres sin } \\
\text { cáncer }\end{array}$} \\
\hline & & $\alpha$ & $M$ & $D T$ & $M$ & $D T$ & $M$ & $D T$ \\
\hline Edad & $25-69$ & & $\begin{array}{c}44.2 \\
7\end{array}$ & 11.80 & 52.20 & 8.85 & 38.78 & 10.41 \\
\hline $\begin{array}{c}\text { ResPro } \\
\text { bl }\end{array}$ & $2-20$ & .82 & $\begin{array}{c}15.4 \\
2\end{array}$ & 4.01 & 15.60 & 4.00 & 15.30 & 4.06 \\
\hline$\underset{t}{\text { AutoCri }}$ & $0-20$ & .87 & 6.81 & 5.82 & 5.84 & 5.24 & 7.49 & 6.14 \\
\hline $\begin{array}{c}\text { ExprE } \\
\text { moc }\end{array}$ & $1-20$ & .78 & $\begin{array}{c}11.8 \\
4\end{array}$ & 5.06 & 10.51 & 5.20 & 12.77 & 4.79 \\
\hline $\begin{array}{l}\text { PensDes } \\
\text { id }\end{array}$ & $3-20$ & .77 & $\begin{array}{c}15.3 \\
6\end{array}$ & 4.20 & 15.24 & 4.17 & 15.44 & 4.25 \\
\hline $\begin{array}{l}\text { ApoyoS } \\
\text { oc }\end{array}$ & $0-20$ & .84 & $\begin{array}{c}14.2 \\
4\end{array}$ & 4.88 & 13.95 & 4.72 & 14.44 & 5.03 \\
\hline $\begin{array}{c}\text { ReestrC } \\
\text { og }\end{array}$ & $1-20$ & .79 & $\begin{array}{c}11.8 \\
3\end{array}$ & 4.80 & 12.17 & 4.70 & 11.60 & 4.89 \\
\hline $\begin{array}{c}\text { EvitPro } \\
\text { bl }\end{array}$ & $0-20$ & .75 & 6.51 & 4.74 & 6.89 & 4.77 & 6.26 & 4.75 \\
\hline $\begin{array}{l}\text { RetirSo } \\
\text { c }\end{array}$ & $0-20$ & .79 & 6.75 & 5.48 & 6.46 & 5.49 & 6.95 & 5.50 \\
\hline CPT & $\begin{array}{l}14- \\
105\end{array}$ & .92 & 74.3 & 19.48 & 76.66 & $\begin{array}{c}18.8 \\
8\end{array}$ & 72.66 & 20.31 \\
\hline
\end{tabular}

Nota. ResProbl=Resolución de Problemas; AutoCrit= Autocrítica; ExprEmoc $=$ Expresión de emociones; PensDesid $=$ Pensamiento Desiderativo; ApoyoSoc $=$ Apoyo Social; ReestruCog $=$ Reestructuración Cognitiva; EvitProbl=Evitación de Problemas; RetirSoc $=$ Retirada Social; $C P T=$ Crecimiento Postraumático.

Para comprobar si las diferencias encontradas anteriormente entre ambos grupos son estadísticamente significativas y poder dar respuesta al primer objetivo, se llevó a cabo un análisis de varianza ANOVA (Ver tabla 2). Los resultados nos indican que únicamente se encuentran diferencias intergrupales estadísticamente significativas en la estrategia expresión de emociones. A la luz de los datos obtenidos se puede inferir que las mujeres con cáncer de mama hacen menor uso de la estrategia expresión de emociones que aquellas que no padecen esta enfermedad, como indican los datos descriptivos de la Tabla 1, donde la media de la puntuación de dicha estrategia en mujeres de cáncer de mama ha sido 10.51 y en mujeres sin cáncer 12.77 . 
Tabla 2. Análisis ANOVA de las estrategias de afrontamiento y el CPT

\begin{tabular}{|c|c|c|c|c|c|c|}
\hline & & $\begin{array}{c}\text { Suma de } \\
\text { cuadrados }\end{array}$ & gl & $\begin{array}{c}\text { Media } \\
\text { cuadrática }\end{array}$ & $\mathbf{F}$ & Sig. \\
\hline \multirow{3}{*}{$\begin{array}{l}\text { Resolución de } \\
\text { problemas }\end{array}$} & Intergrupos & 2,272 & 1 & 2,272 &, 140 &, 709 \\
\hline & Intragrupos & 1758,646 & 108 & 16,284 & & \\
\hline & Total & 1760,918 & 109 & & & \\
\hline & Intergrupos & 72,206 & 1 & 72,206 & 2,151 & 145 \\
\hline \multirow[t]{2}{*}{ Autocrítica } & Intragrupos & 3626,157 & 108 & 33,576 & & \\
\hline & Total & 3698,364 & 109 & & & \\
\hline \multirow{3}{*}{$\begin{array}{l}\text { Expresión de } \\
\text { emociones }\end{array}$} & Intergrupos & 135,590 & 1 & 135,590 & 5,512 &, 021 \\
\hline & Intragrupos & 2656,783 & 108 & 24,600 & & \\
\hline & Total & 2792,373 & 109 & & & \\
\hline \multirow{4}{*}{$\begin{array}{l}\text { Pensamiento } \\
\text { desiderativo }\end{array}$} & Intergrupos & 1,082 & 1 & 1,082 &, 061 & ,806 \\
\hline & Intragrupos & 1924,373 & 108 & 17,818 & & \\
\hline & Total & 1925,455 & 109 & & & \\
\hline & Intergrupos & 6,400 & 1 & 6,400 & ,266 & ,607 \\
\hline \multirow[t]{2}{*}{ Apoyo Social } & Intragrupos & 2599,973 & 108 & 24,074 & & \\
\hline & Total & 2606,373 & 109 & & & \\
\hline \multirow{3}{*}{$\begin{array}{c}\text { Reestructuración } \\
\text { cognitiva }\end{array}$} & Intergrupos & 8,877 & 1 & 8,877 & ,383 &, 537 \\
\hline & Intragrupos & 2504,178 & 108 & 23,187 & & \\
\hline & Total & 2513,055 & 109 & & & \\
\hline \multirow{3}{*}{$\begin{array}{l}\text { Evitación de } \\
\text { problemas }\end{array}$} & Intergrupos & 10,465 & 1 & 10,465 & ,462 & ,498 \\
\hline & Intragrupos & 2446,998 & 108 & 22,657 & & \\
\hline & Total & 2457,464 & 109 & & & \\
\hline \multirow{3}{*}{ Retirada Social } & Intergrupos & 6,311 & 1 & 6,311 & ,208 & ,649 \\
\hline & Intragrupos & 3272,062 & 108 & 30,297 & & \\
\hline & Total & 3278,373 & 109 & & & \\
\hline \multirow{3}{*}{$\begin{array}{l}\text { Crecimiento } \\
\text { Postraumático }\end{array}$} & Intergrupos & 426,546 & 1 & 426,546 & 1,125 & ,291 \\
\hline & Intragrupos & 40956,554 & 108 & 379,227 & & \\
\hline & Total & 41383,100 & 109 & & & \\
\hline
\end{tabular}

Para dar respuesta a nuestro segundo objetivo se realizó un análisis correlacional entre las diferentes estrategias de afrontamiento y el Crecimiento Postraumático (ver Tabla 3). Los resultados nos advierten que el Crecimiento Postraumático mantiene una relación positiva estadísticamente significativa con las estrategias resolución de problemas, expresión de emociones, apoyo social y reestructuración cognitiva. Además, existen relaciones significativas desde un punto de vista estadístico entre las diferentes estrategias de afrontamiento. 
Tabla 3. Análisis de correlación bivariada entre estrategias de afrontamiento y CPT.

\begin{tabular}{|c|c|c|c|c|c|c|c|c|c|}
\hline & CPT & 1 & 2 & 3 & 4 & 5 & 6 & 7 & 8 \\
\hline $\begin{array}{l}\text { 1. Resolución de } \\
\text { problemas }\end{array}$ & $.380 * *$ & - & - & - & - & - & - & - & - \\
\hline 2. Autocrítica & -.152 & $\begin{array}{l}.296 \\
* *\end{array}$ & & & & & & & \\
\hline $\begin{array}{l}\text { 3. Expresión de } \\
\text { emociones }\end{array}$ & $.295 * *$ & $\underset{* *}{.322}$ & $\begin{array}{c}- \\
.084\end{array}$ & - & - & - & - & - & - \\
\hline $\begin{array}{l}\text { 4. Pensamiento } \\
\text { desiderativo }\end{array}$ & .085 & -.079 & $\underset{* *}{.292}$ & .175 & - & - & - & - & - \\
\hline 5. Apoyo social & $.424 * *$ & $\begin{array}{l}.488 \\
* *\end{array}$ & $\begin{array}{l}- \\
.210 \\
*\end{array}$ & $\begin{array}{c}.542 * \\
*\end{array}$ & .171 & - & - & - & - \\
\hline $\begin{array}{l}\text { 6. Reestructuraci } \\
\text { ón cognitiva }\end{array}$ & $.322 * *$ & $\begin{array}{l}.450 \\
* *\end{array}$ & $\begin{array}{c}- \\
.194 \\
*\end{array}$ & $.201 *$ & - & $\begin{array}{c}.424 * \\
*\end{array}$ & - & - & - \\
\hline $\begin{array}{l}\text { 7. Evitación de } \\
\text { problemas }\end{array}$ & -.104 & -.019 & $\begin{array}{c}- \\
.020\end{array}$ & -.074 & .002 & -.018 & $\begin{array}{c}.442 \\
* *\end{array}$ & - & - \\
\hline 8. Retirada social & -.113 & $\begin{array}{c}- \\
.241 \\
*\end{array}$ & $\begin{array}{c}.554 \\
*\end{array}$ & $\begin{array}{c}- \\
.369 * \\
*\end{array}$ & .177 & $\begin{array}{c}- \\
.496 * \\
*\end{array}$ & $\begin{array}{c}- \\
.196 \\
*\end{array}$ & $\begin{array}{c}.12 \\
4\end{array}$ & - \\
\hline
\end{tabular}

Por último, a fin de dar respuesta a nuestro tercer objetivo comprobar la capacidad explicativa y predictiva de las estrategias de afrontamiento sobre el Crecimiento Postraumático, realizamos un análisis de regresión lineal por pasos, tomando como variables predictoras las estrategias de afrontamiento que mostraron una relación estadísticamente significativa con la CPT, y como variable dependiente el CPT., controlamos el efecto de la edad y el padecer, o no, cáncer, del cual se extrajeron dos modelos estadísticamente significativos (ver Tabla 4).

Tabla 4. Análisis de regresión lineal por pasos

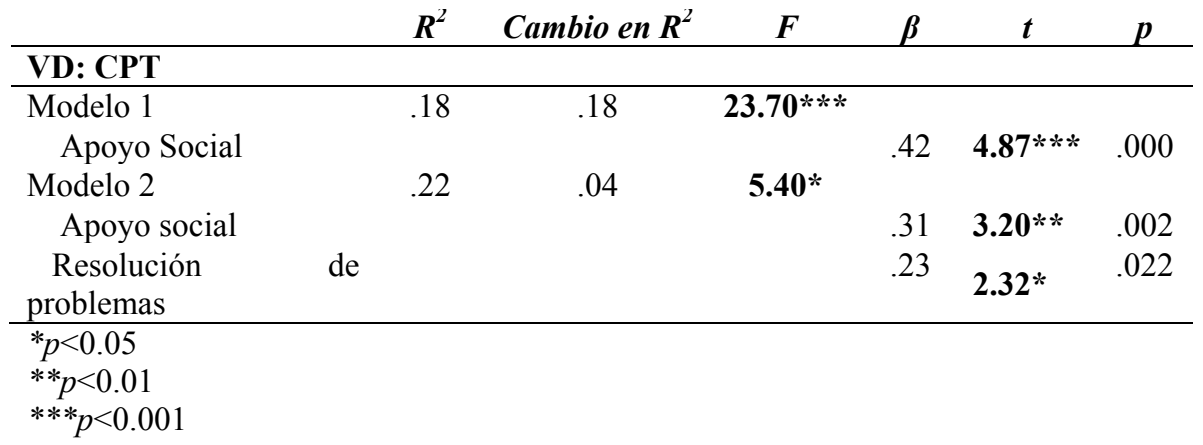


En el primer modelo estadísticamente significativo, el Apoyo Social explica un 18\% de la varianza del CPT $\left(R^{2}=, 180\right)$. En el segundo, al Apoyo Social se le añade la Resolución de Problemas, explicando ambos un $21,9 \%\left(R^{2}=, 219\right)$ de la varianza del CPT. Ambos modelos muestran, además, una capacidad predictiva estadísticamente significativa sobre el CPT. Estos resultados se muestran de manera visual en los Gráficos 1 y 2.

Gráfico 1. Capacidad explicativa y predictiva del Modelo 1.

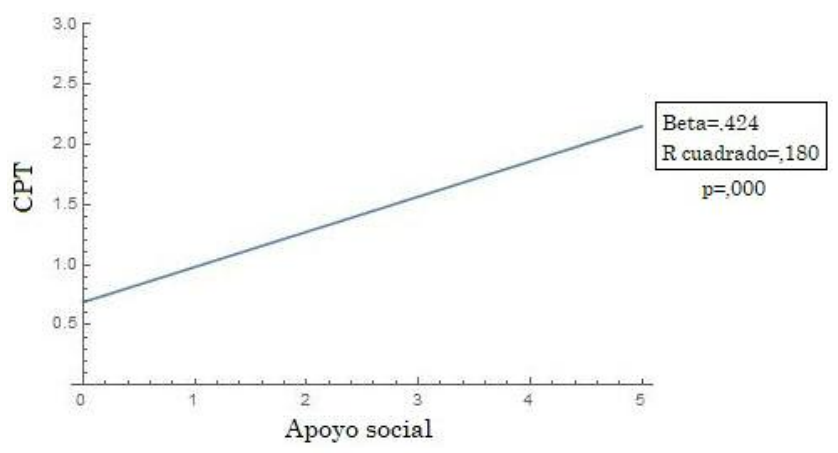

Gráfico 2. Capacidad explicativa y predictiva del Modelo 2.

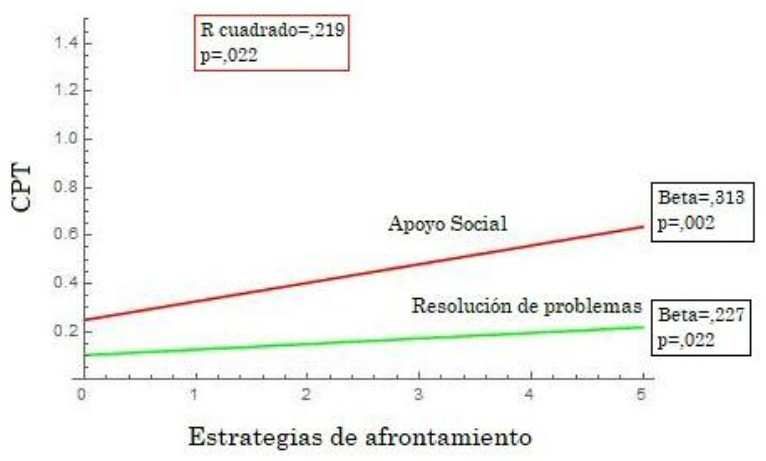

Se deduce, de este modo, que tanto el Apoyo Social como la Resolución de Problemas poseen una capacidad tanto explicativa como predictiva del Crecimiento Postraumático de estas 110 mujeres, con independencia de la edad de las mismas y de haber padecido cáncer de mama o no.

\section{DISCUSIÓN}

Si bien los datos descriptivos nos indican que las mujeres con cáncer presentan una media mayor en el CPT que las mujeres que no padecen esta enfermedad, los resultados obtenidos nos advierten que no existen diferencias estadísticamente significativas en el CPT de ambos grupos. Eso se traduce en que estas mujeres presentan niveles similares en su Crecimiento Postraumático, ya sea obtenido a raíz de la experiencia de padecer cáncer o de sufrir cualquier otro evento traumático 
0 estresante. Estamos de acuerdo con Zayas et al. (2018) en que el hecho de padecer cáncer de mama, la incertidumbre del pronóstico, los efectos secundarios de los tratamientos y los cambios en la vida cotidiana derivados del diagnóstico hacen que las mujeres que lo vivencian activen un mecanismo de crecimiento postraumático. No obstante, podemos confirmar que esto mismo ocurre en el caso de otras mujeres que hayan acontecido otro tipo de situaciones traumáticas y adversas.

Igualmente, encontramos que tampoco existen diferencias estadísticamente significativas entre las distintas estrategias de afrontamiento de ambos grupos, excepto en la expresión de emociones. Atendiendo a los datos descriptivos, apreciamos que las mujeres con cáncer presentan una media inferior que las mujeres sin cáncer en esta variable. Ello quiere decir que las mujeres que padecen esta enfermedad hacen menor uso de la expresión de emociones que aquellas que no han experimentado esta situación. Esto va en consonancia con lo aportado por Cerezo, Ortiz-Tallo y Cardenal (2009), quienes indican que la represión emocional o la dificultad para expresar emociones es común en mujeres con cáncer de mama. Por otra parte, debemos indicar que nuestros resultados no coinciden con lo aportado por De Haro-Rodríguez et al., (2014), quienes indican que las estrategias de afrontamiento más utilizadas por mujeres con cáncer de mama son la resolución de problemas, apoyo social y el pensamiento desiderativo. Según nuestros análisis, aquellas más usadas por estas mujeres, en comparación con mujeres que no presentan esta enfermedad, son la resolución de problemas, la reestructuración cognitiva y la evitación de problemas.

Al no encontrar diferencias significativas entre los grupos, nos planteamos la necesidad de investigar sobre la relación entre las distintas estrategias de afrontamiento y el CPT y si ésta está influida por la presencia del cáncer y la edad de estas mujeres. Se evidencian, en este sentido, relaciones entre el CPT y las estrategias de afrontamiento resolución de problemas, expresión de emociones, apoyo social y reestructuración cognitiva. Ello coincide con lo indicado por Prati y Pietrantoni (2009), para quienes tanto las estrategias de afrontamiento como el apoyo social contribuyen al crecimiento obtenido tras un hecho traumático.

Los resultados obtenidos confirman la relevancia del uso de estrategias eficaces como la búsqueda de apoyo emocional y la resolución de problemas en el proceso de CPT, descartando la influencia del cáncer y la edad. Especialmente, el apoyo social se constituye como la variable con mayor influencia sobre el CPT, seguida de la resolución de problemas. Ambas variables presentan capacidad explicativa y predictiva de este crecimiento. Estamos de acuerdo con el modelo de Schaefer y Moos (1998) que considera el apoyo social un recurso ambiental clave para comprender resultados positivos de crisis vitales, así como con el de Tedeschi y Calhoun (2004), que incluye el apoyo social como predictor de cambios positivos tras eventos traumáticos. Además, ya que en nuestro estudio el apoyo social es considerado como una estrategia de búsqueda de apoyo emocional (Cano y cols., 2007), también podemos confirmar que existen evidencias de que tanto el apoyo social como la búsqueda de apoyo pueden promover el CPT (Schaefer y Moos, 1998; Tedeschi y Calhoun, 2004).

\section{CONCLUSIONES}

A la luz de los resultados obtenidos mediante el presente estudio se puede concluir que:

Las mujeres que han padecido cáncer de mama no presentan diferencias estadísticamente significativas respecto de aquellas que no han experimentado ésta situación en su CPT ni en las estrategias de afrontamiento, excepto en la estrategia expresión de emociones cuyo uso es menos frecuente en las mujeres con cáncer.

EI CPT está relacionado de manera estadísticamente significativa con las estrategias resolución 


\section{ESTRATEGIAS DE AFRONTAMIENTO Y CRECIMIENTO POSTRAUMÁTICO EN MUJERES CON Y SIN CÁNCER DE MAMA}

de problemas, la expresión de emociones, el apoyo social y la reestructuración cognitiva. Asimismo, existen relaciones con significación estadística entre las distintas estrategias de afrontamiento.

El padecimiento de cáncer de mama y la edad no es un factor explicativo ni predictivo del Crecimiento Postraumático. Si bien, las estrategias apoyo social y resolución de problemas explican y predicen este fenómeno.

Potenciar el apoyo social y la resolución de problemas podría actuar como estrategia protectora del CPT de mujeres, ya sea en poblaciones clínicas como no clínicas.

Asimismo, sería adecuado trabajar sobre la estrategia expresión de emociones, así como con aquellas que mantienen una relación estadísticamente significativa con la misma. En este sentido, la expresión de emociones correlaciona positivamente con el apoyo social y la resolución de problemas. Por tanto, promover la expresión de emociones potenciaría ambas estrategias y, por ende, mejoraría el CPT, el bienestar y la calidad de vida de las mujeres.

De cara a estudios futuros, sería interesante incluir las subdimensiones del CPT, ya que podrían hallarse resultados que en esta investigación pasan desapercibidos.

\section{REFERENCIAS BIBLIOGRÁFICAS}

Acinas, M. P. (2014). Situaciones traumáticas y resiliencia en personas con cáncer. Psiquiatría biológica, 21(2), 65-71.

Avilés, P., Cova, F., Bustos, C. \& García, F. (2014). Afrontamiento y rumiación frente a eventos adversos y crecimiento postraumático en estudiantes universitarios. Liberabit, 20(2), 281-292.

Barroilhet, S., Forjaz, M. J., \& Garrido, E. (2005). Conceptos, teorías y factores psicosociales en la adaptación al cáncer. Actas españolas de psiquiatría, 33(6).

Calhoum L.G. \& Tedeschi R.G. The Foundations of Posttraumatic Growth: an expanded framework. En: Calhoum L.G. \& Tedeschi R.G. (editors) (2006). Handbook of Posttraumatic Growth. Mahwah, NJ: Lawrence Erlbaum Associate Publishers; p. 3-23.

Campos-Ríos, M. D. M. (2013). Creciendo en la adversidad: Una revisión del proceso de adaptación al diagnóstico de cáncer y el crecimiento postraumático. Escritos de Psicología, 6(1), 6-13.

Cano, A. (2005). Control emocional, estilo represivo de afrontamiento y cáncer: ansiedad y cáncer. Psicooncología, 2(1), 71-80.

Cano, G.F., Rodríguez, F.L. \& García, M.J. (2007). Adaptación española del Inventario de Estrategias de Afrontamiento. Actas Españolas de Psiquiatría, 35(1), 29-39.

Castaño, E. F., \& León del Barco, B. (2010). Estrategias de afrontamiento del estrés y estilos de conducta interpersonal. International Journal of psychology and psychological therapy, 10(2).

Cerezo, M. V., Ortiz-Tallo, M., \& Cardenal, V. (2009). Expresión de emociones y bienestar en un grupo de mujeres con cáncer de mama: una intervención psicológica. Revista Latinoamericana de Psicología, 41(1), 129-138.

Costa, G., \& Gil, F. L. (2007). Crecimiento postraumático en pacientes oncológicos. Análisis y modificación de conducta, 33(148).

Costa, G., \& Gil, F. L. (2008). Respuesta cognitiva y crecimiento postraumático durante el primer año de diagnóstico del cáncer. Psicooncología, 5(1).

De Haro-Rodríguez, M. A., Gallardo-Vid, L. S., Martínez, M. L. M., Camacho-Calderón, N., VelázquezTlapanco, J., \& Hernández, E. P. (2014). Factores relacionados con las diferentes estrategias de afrontamiento al cáncer de mama en pacientes de recién diagnóstico. Psicooncología, 11(1), 8799.

Fierro A. (1993). Para una ciencia del sujeto: Investigación de la personalidad. Barcelona: Anthropos. Guil, R., Zayas, A., Gil-Olarte, P., Guerrero, C. \& Mestre, J. M. (2017). Ejercicio físico y aspectos psi- 
cosociales y emocionales en mujeres con cáncer de mama. International Journal of Development and Educational Psychology. Revista INFAD de Psicología, 4(1), 327-338.

Guil, R., Zayas, A., Gil-Olarte, P., Guerrero, C., González, S. \& Mestre, J. M. (2016). Bienestar psicológico, optimismo y resiliencia en mujeres con cáncer de mama. Psicooncología, 13(1), 127.

Guzmán, M. V. (2014). Variables psicológicas positivas en pacientes con cáncer. Informació Psicólogica, 106, 17-27.

Lazarus, R. S., \& Folkman, S. (1984). Stress, appraisal, and coping. New York: Springer.

Nava-Quiroz, C., Ollua, P., Vega, C. \& Soria, R. (2010). Inventario de Estrategias de Afrontamiento: Una replicación. Psicología y Salud, 20 (2), 213-220.

Ochoa, C., Castejón, V., Sumalla, E. C., \& Blanco, I. (2013). Crecimiento Post-traumático en supervivientes de cáncer y sus otros significativos: ¿Crecimiento vicario o secundario?. Terapia psicológica, 31(1), 81-92.

Prati, G., \& Pietrantoni, L. (2009). Optimism, social support, and coping strategies as factors contributing to posttraumatic growth: A meta-analysis. Journal of loss and trauma, 14(5), 364-388.

Schaefer, J. A., \& Moos, R. H. (1998). The context for posttraumatic growth: Life crises, individual and social resources, and coping. Posttraumatic growth: Positive changes in the aftermath of crisis, 99, 126.

Sociedad Española de Oncología Médica (SEOM) (2016). Las cifras del Cáncer en España 2014. Madrid.

Tedeschi, R.G., Calhoum, L.G. (1996). The posttraumatic Growth Inventory: Measuring the Positive Legacy of Trauma. Trauma Stress, 9(3), 455-71.

Tedeschi, R. G., \& Calhoun, L. G. (1995). Trauma and transformation. Sage.

Tedeschi, R. G., \& Calhoun, L. G. (2004). Posttraumatic growth: Conceptual foundations and empirical evidence. Psychological inquiry, 15(1), 1-18.

Tobin, D. L., Holroyd, K. A., Reynolds, R. V., \& Wigal, J. K. (1989). The hierarchical factor structure of the Coping Strategies Inventory. Cognitive therapy and research, 13(4), 343-361.

Vázquez, C., \& Castilla, C. (2007). Emociones positivas y crecimiento postraumático en el cáncer de mama. Psicooncología, 4(2/3), 385.

Vera, B., Carbelo, B., \& Vecina, M. L. (2006). La experiencia traumática desde la psicología positiva: resiliencia y crecimiento postraumático. Papeles del psicólogo, 27(1).

Zayas, A., Gómez-Molinero, R., Guil, R., Gil-Olarte, P. \& Jiménez, E. (2018). Relación entre la resiliencia y la satisfacción con la vida en una muestra de mujeres con cáncer de mama. International Journal of Developmental and Educational Psychology. Revista de Psicología INFAD, 1(2), 127-136. 
